\title{
An Anomalous Conductance Decrease in Charge Sensitive Infrared Phototransistor
}

\author{
Ting-Ting Kang, Susumu Komiyama, Takeji Ueda, Shi-Wei Lin, and Sheng-Di Lin
}

\begin{abstract}
For charge-sensitive infrared phototransistors (CSIP), it is observed that "conductance decrease," which is contrary to the standard "conductance increase" photon response, can also happen after absorbing infrared light. By experimental modeling the charge-up detection mechanism of CSIP via a capacitive way, we clarify that "conductance decrease" should be attributed to the significantly reduced low quantum well electron mobility after the photon-charging process, rather than a reversed electron transfer. This experimental result clearly indicates that photon-induced charges are able to modify the electron mobility in those "charge-sensitive sensor" types of semiconductor quantum single-photon detectors.
\end{abstract}

Index Terms-Charge, detector, double quantum well, infrared, mobility, phototransistor.

\section{INTRODUCTION}

$\mathbf{T}$ HE single-photon detection in the long-wavelength range $(\lambda>10 \mu \mathrm{m})$ is an important field, where only semiconductor quantum devices are the mature detectors available [1]. These single-photon detectors, like quantum dot (QD) detectors [2], are based on a charge-sensitive sensor scheme [1], in which the active region is polarized by photoexcitation and the induced polarization is sensed by a nearby charge-sensitive sensor.

A custom-friendly infrared photon detector operating at $4.2 \mathrm{~K}$ has been developed recently, which is called chargesensitive infrared phototransistors (CSIP) [1], [3]-[7]. CSIP detector utilizes double quantum well (DQW) structures, in which an isolated top QW island (TQWI) serves as the active region and a separated conductive channel consisting of the low QW functions as the sensor. The operation mechanism of CSIP has

Manuscript received January 5, 2012; revised February 28, 2012; accepted March 15, 2012. Date of publication March 22, 2012; date of current version February 1, 2013. This work was supported by the Core Research for Evolutional Science and Technology (CREST) Project of the Japan Science and Technology Agency.

T.-T. Kang is with the Department of Basic Science, The University of Tokyo, Tokyo 153-8902, Japan and also with the Japan Science and Technology Corporation, Saitama 332-0012, Japan and also with the Shanghai Institute of Technical Physics, Chinese Academy of Sciences, Shanghai 200083, China (e-mail: ktt219@163.com).

S. Komiyama is with the Department of Basic Science, The University of Tokyo, Tokyo 153-8902, Japan.

T. Ueda is with the Department of Basic Science, The University of Tokyo, Tokyo 153-8902, Japan and also with the Japan Science and Technology Corporation, Saitama 332-0012, Japan.

S.-W. Lin and S.-D. Lin are with the Department of Electronics Engineering, National Chiao Tung University, Hsinchu 300, Taiwan.

Color versions of one or more of the figures in this paper are available online at http://ieeexplore.ieee.org.

Digital Object Identifier 10.1109/JSTQE.2012.2191767

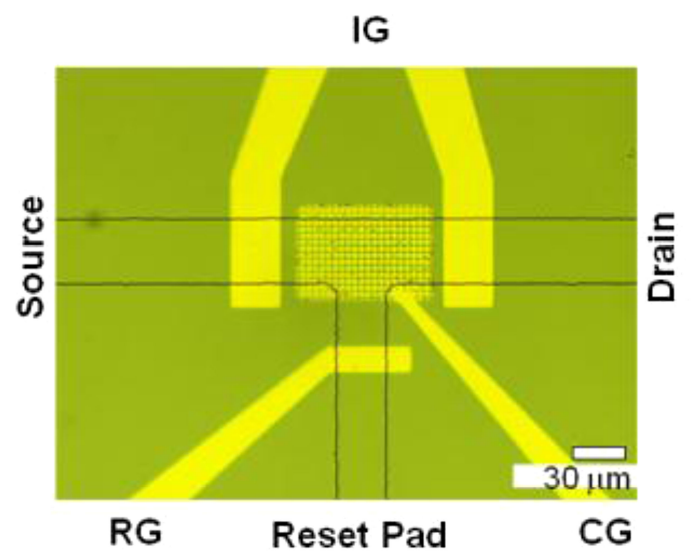

(a)

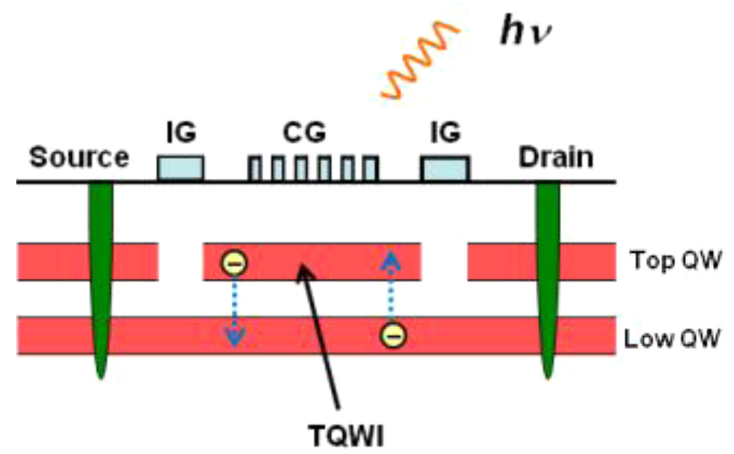

(b)

Fig. 1. (a) Optical image of the CSIP device used here; (b) schematic illustration of infrared light excited electron transfer in CSIP. In (b), left/right side, a "top-to-low"/"low-to-top" electron transfer is plotted. And "top-to-low" is the standard CSIP detecting mechanism.

been widely documented. That is, after infrared (IR) illumination, the electrons excited by intersubband transition (ISBT) in TQWI move to the low QW layer after tunneling out of top QW. Missing electrons, TQWI is positively charged up, which in turn increases the conductance of low QW layer (so it is named as "Conductance Increase") by enhancing its electron density in a capacitive way. This "top-to-low" electron transfer is schematic drawn in Fig. 1(b), left part of TQWI.

"Conductance Increase" has been observed in CSIP for many times and become a trivial thing. Because people are so satisfied with the agreement between "Conductance Increase" and the given interpretation, it is inconceivable that "Conductance Decrease" can also appear in some CSIPs. A naive way to explain "Conductance Decrease" has been tried in [7], which claimed that "Conductance Decrease" may be due to a reversed electron transfer. Adopting this picture for CSIP with vertical 
tunneling, it means a "low-to-top" electron transfer: electrons downside TQWI are excited by incoming light and flow into TQWI (see Fig. 1(b), right part of TQWI), leaving TQWI negatively charged and reducing the electron density in low QW. What we seriously concern is that "Conductance Decrease" may compensate "Conductance Increase" to some extent, so that degenerate the performance of CSIP. In a more fundamental view, "Conductance Decrease" can be used to challenge our previous interpretation of CSIP operation. Therefore, clarifying the mechanism of "Conductance Decrease" is critical for CSIP and will be addressed in this paper.

\section{DEVICE STRUCTURE AND FABRICATION}

We first specify the device structures and measurement conditions. Two AlGaAs/GaAs DQW wafers (labeled as Wafers "A" and "B") are grown by molecular-beam expitaxy on an insulative GaAs substrate. The designed structures are the same for these two wafers. It consist of a 500-nm GaAs buffer layer, a 210-nm-thick AlGaAs/AlAs superlattice barrier, a 20-nm ndoping ( $\mathrm{Si}: 8 \times 10^{17} \mathrm{~cm}^{-3}$ ) $\mathrm{Al}_{0.3} \mathrm{Ga}_{0.7}$ As electron supplier, a 20-nm $\mathrm{Al}_{0.3} \mathrm{Ga}_{0.7}$ As spacer, a 30-nm nondoped GaAs QW (i.e. low QW), an 80-nm composition-graded $\mathrm{Al}_{x} \mathrm{Ga}_{1-x} \mathrm{As}(\mathrm{x}=0 \rightarrow$ 0.1 ) barrier layer, a 2-nm $\mathrm{Al}_{0.2} \mathrm{Ga}_{0.8}$ As tunnel barrier, a 10-nm GaAs QW layer (i.e., top QW), a 30-nm $\mathrm{Al}_{0.3} \mathrm{Ga}_{0.7}$ As spacer, a 75-nm n-doping ( $\mathrm{Si}: 8 \times 10^{17} \mathrm{~cm}^{-3}$ ) $\mathrm{Al}_{0.3} \mathrm{Ga}_{0.7}$ As electron supplier, and a 5 -nm n-doping (Si: $8 \times 10^{17} \mathrm{~cm}^{-3}$ ) GaAs cap layer.

We have fabricated the devices on both wafers with the same procedure indicated later. Fig. 1(a) shows the device layout of CSIP used throughout this paper. A two-terminal conductor with a constricted region of a $50 \mu \mathrm{m}$ width and a $400 \mu \mathrm{m}$ length is formed by wet mesa etching with $\sim 200 \mathrm{~nm}$ depth. Ohmic contacts (source, drain, and reset pad), which penetrate both top and low QW [see Fig. 1(b)], are prepared by alloying of a 200-nm-thick Au-Ge 10\%-Ni 4\% layer. The Schottky contacts, including isolation gate (IG), reset gate (RG), and coupler gate (CG), are formed by depositing a metal layer $20 \mathrm{~nm} \mathrm{Ti} / 100 \mathrm{~nm}$ $\mathrm{Au}$. The roles of reset pad and $\mathrm{RG}$ are controllable releasing of excessive charges in TQWI [6]. To improve the detector's quantum efficiency, both the pattern (square cross-hole arrays) and size (4 $\mu \mathrm{m}$ period) of CG use the suggested ones from [8]. All the measurements were done in $4.2 \mathrm{~K}$ using liquid helium. The $300 \mathrm{~K}$ blackbody radiation from room temperature optical components is transmitted by a cold metal (steel) pipeline and used as an excitation light source. The source-drain bias voltage is maintained as $V_{\mathrm{DS}}=8.5 \mathrm{mV}(8.2 \mathrm{mV})$ for the "Conductance Increase"("Conductance Decrease") CSIP.

\section{RESUlTS AND DISCUSSION}

Following the method in [3], the photon-induced conductance variations can be seen from the plot of source drain current $I_{\mathrm{DS}}$ against IG gate voltage $V_{\mathrm{IG}}$, as shown in Fig. 2. In this measurement, CG just acts as an antenna to couple light with ISBT and plays no other roles (like electric manipulation), so its voltage $V_{\mathrm{CG}}=0 \mathrm{~V}$. Before the $I_{\mathrm{DS}}$ versus $V_{\mathrm{IG}}$ mea-

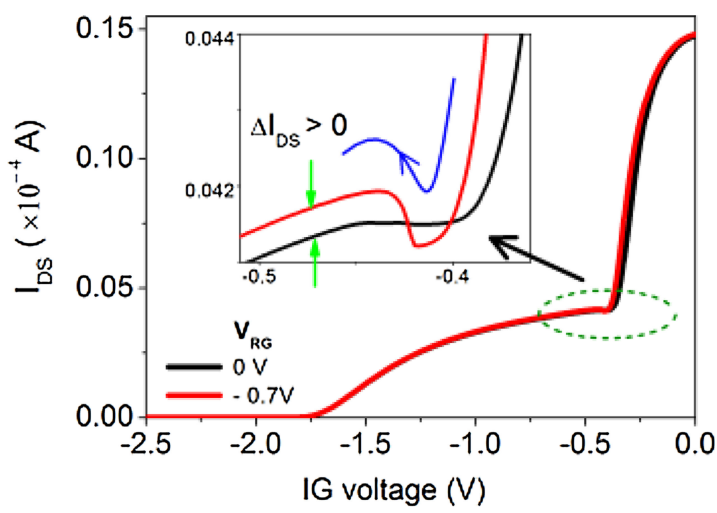

(a)

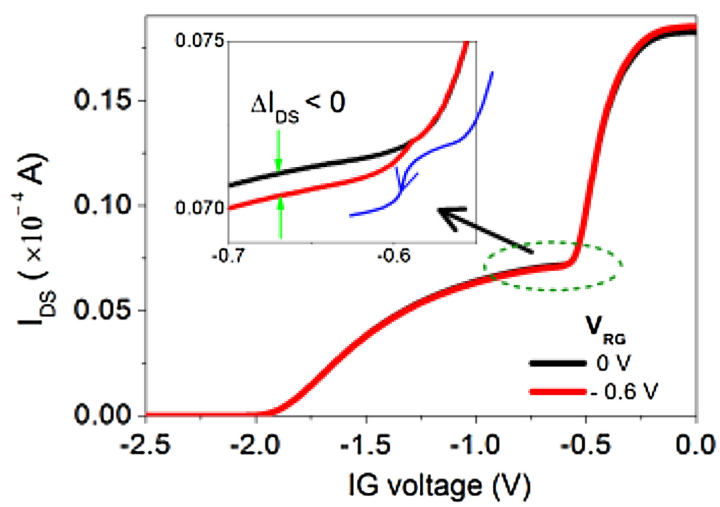

(b)

Fig. 2. $I_{\mathrm{DS}}$ against $V_{\mathrm{IG}}$ plot of CSIP with a photon response of (a) Wafer A with "Conductance Increase"; (b) Wafer B with "Conductance Decrease." Inset: magnified view of the "kink" features in the region near the top QW cut-off voltage $V_{g}$. The blue schematic curve (with arrow) inset is a cartoon drawing showing where the increase/decrease structures of the "kink" are presented.

surement, a voltage $V_{\mathrm{RG}}$ is applied to RG to deplete top QW in the reset route. Thus, $V_{\mathrm{RG}}$ should meet the request $V_{\mathrm{RG}}=$ $V_{1}<V_{g 1}\left(V_{g 1} / V_{g 2}\right.$ : the depleting voltage of top/low QW). From Fig. 1, it is shown that $V_{g 1} \sim-0.42 \mathrm{~V}[-0.59 \mathrm{~V}]$, so that $V_{1}=$ $-0.7 \mathrm{~V}[-0.6 \mathrm{~V}]$ in Fig. 2(a) [Fig. 2(b)] is adopted, respectively.

In a capacitor configuration, the area electron density $n$ in each QW layer can be deduced by $n \approx \Delta \mathrm{V} \varepsilon_{0} \varepsilon_{r} /(d e)\left(\varepsilon_{r}\right.$ : dielectric constant of semiconductor; $\varepsilon_{0}$ : vacuum permittivity; $d$ : the distance between the metal gate and the desired QW; $e$ : charge of a free electron), where $\Delta V=\left(0-V_{g 1}\right)\left[\left(V_{g 1}-V_{g 2}\right)\right]$ for top [low] QW. Then with the conductivity deduced from dc measurement, the electron mobility $\mu$ in each QW layer can be calculated. In this way, we get the electron concentration $\left(\times 10^{11}\right.$ $\left.\mathrm{cm}^{-2}\right)$ and mobility $\left(\times 10^{4} \mathrm{~cm}^{2} \mathrm{~V}^{-1} \mathrm{~s}^{-1}\right)$ for top/low $\mathrm{QW}$ at 4.2 K: 2.7/4.8 and 23.0/5.0 for Wafer A; 3.6/4.8 and 19.0/9.0 for Wafer B.

For both devices, the $I_{\mathrm{DS}}$ versus $V_{\mathrm{IG}}$ plot (the $V_{\mathrm{RG}}=-0.7 \mathrm{~V}$, $-0.6 \mathrm{~V}$ plot) shows a kink (schematic drawn in the blue curve with an arrow) at $V_{g 1}$, where top QW is depleted in the IG region. Such kink features are attributed to the photon-induced charging of TQWI. This is supported by the fact that if $V_{\mathrm{RG}}=0 \mathrm{~V}$, for such case TQWI is not isolated and cannot be charged up, those kink features disappear (see the $V_{\mathrm{RG}}=0 \mathrm{~V}$ plots). Regarding Wafer 
A in Fig. 2(a), with the standard "Conductance Increase" photon response in CSIP, the "rising-up" (See the inset, the direction of an arrow in schematic curve shows the "rising") kink is well understood as the positively charging up of TQWI by photons. Consequently, when $V_{\mathrm{IG}}<-0.42 \mathrm{~V}$, a low $\mathrm{QW}$ conductance increase leads to $\Delta I_{\mathrm{DS}}=I_{\mathrm{DS}}\left(V_{\mathrm{RG}}=-0.7 \mathrm{~V}\right)-I_{\mathrm{DS}}\left(V_{\mathrm{RG}}=\right.$ $0 \mathrm{~V})>0$, as indicated by the straight green arrows (in a vertical direction) in Fig. 2(a).

However, the kink for Wafer B in Fig. 2(b) is some strange. It displays a "turn-down" trend. Compared with the $I_{\mathrm{DS}}$ versus $V_{\mathrm{IG}}$ plot with $V_{\mathrm{RG}}=0 \mathrm{~V}$, a reduction of $I_{\mathrm{DS}}$ (i.e., $\Delta I_{\mathrm{DS}}<0$ ) is clearly present when top QW is depleted for $V_{\mathrm{IG}}<-0.59 \mathrm{~V}$. And this low QW conductance decrease is just the "Conductance Decrease" problem mentioned in the beginning.

One first possible explanation of "Conductance Decrease" might be to assume the opposite process to the standard "top-tolow" mechanism, i.e., "low-to-top" tunneling: low QW is somehow photon-excited and the photon-excited electrons move to TOWI. While, as discussed later, this simple interpretation is not likely to be applicable. Because it needs an unreasonable assumption: wide QW (i.e., low QW) has a higher ISBT probability than narrow QW (i.e., top QW).

In a half-quantitative way, the probability $p_{\mathrm{T} \rightarrow \mathrm{L}}$ for an electron transferring from top QW to low QW via ISBT plus tunneling is $p_{\mathrm{T} \rightarrow \mathrm{L}}=\eta_{\mathrm{T}} \cdot T$, where $\eta_{\mathrm{T}}\left(\eta_{\mathrm{L}}\right)$ is the quantum efficiency for ISBT from the ground state 0 in top(low) QW to resonant tunneling level $\gamma$ ( or the first exciting level of the top QW); $T$ is the tunneling probability for the state $\gamma$ across the $\mathrm{AlGaAs}$ inter-QW barrier. $\eta_{\mathrm{T}}\left(\eta_{\mathrm{L}}\right)$ is proportional to top(low) QW intersubband (state $0 \rightarrow$ state $\gamma$ ) oscillator strength $f_{\mathrm{T}}\left(f_{\mathrm{L}}\right)$, i.e., $\eta_{\mathrm{T}} \propto$ $f_{\mathrm{T}}$, so that $p_{\mathrm{T} \rightarrow \mathrm{L}} \propto f_{\mathrm{T}} \cdot T$. Similarly, we also reach $p_{\mathrm{L} \rightarrow \mathrm{T}} \propto f_{\mathrm{L}} \cdot T$.

With $p_{\mathrm{T} \rightarrow \mathrm{L}}$ and $p_{\mathrm{L} \rightarrow \mathrm{T}}$ formula at hand, the comparison of $f$ value is the next job. It is fortunate that the $f$-sum rule sets the sum of oscillator strengths $f$ for all ISBT to be a constant, i.e., $\sum_{j} f_{0 j} \approx m_{0} / m^{*}$ ( $0 j$ means the transition from the ground state 0 to the state $j$ ), where $m^{*}$ is the effective electron mass of conduction band [9]. For the narrow top QW, the quantum confinement effect makes it contain only one bound excited state $\gamma$, so $f_{\mathrm{T}} \approx m_{0} / m^{*}$. However, the wide low QW has much weaker confinement effect and contains not only the excited state $\gamma$, but also many other bound excited states, so that $f_{\mathrm{L}} \ll$ $m_{0} / m^{*}$, leading to $f_{\mathrm{T}} \gg f_{\mathrm{L}}$. Thus, $p_{\mathrm{T} \rightarrow \mathrm{L}} \gg p_{\mathrm{L} \rightarrow \mathrm{T}}$, accounting for the fact that moving out of the TQWI process is dominant for ISBT excited electrons.

Therefore, we prefer to believe that, in case of "Conductance Decrease", a positively charged TQWI can anomalously reduce (Strangely, it is NOT "increase") low QW conductance, despite the enhanced electron density in low QW. Hence, a straightforward way to resolve this issue is making TQWI positively charged, and then measure whether the low QW conductance increases or decreases.

But how to make sure that TQWI is positively charged? Although the charge polarity in TQWI cannot be definitely known for the photon-induced charging process, if the charging process proceeds in a capacitive way, there will be little controversy regarding the charge polarity in TQWI. This is the guideline for our following "capacitive charging" scheme. In this scheme, charging of TQWI is realized by intentionally applying some bias to the CG /TQWI capacitor. To eliminate photon-induced charging, the experiments below were done under dark condition, which was confirmed by the absence of "kink" in $\mathrm{I}_{\mathrm{DS}}$ versus $\mathrm{V}_{\mathrm{IG}}$ measurement.

Next, we explain the details of this "capacitive charging" scheme. For IG, a voltage $V_{\mathrm{IG}}=V_{1}<V_{g 1}$ (here $\mathrm{V}_{1}=-0.7$ $\mathrm{V}$ for both devices) is applied to deplete only the top QW below IG. For RG, a voltage $V_{\mathrm{RG}}=V_{1}+V_{\text {pulse }}$ is applied. $V_{\text {pulse }}$ is a pulse wave with frequency $2 f$, and the pulse amplitude is $-V_{1}$ (See Fig. 3(a) and (b), middle). In the pulse duty time, $V_{\mathrm{RG}}=0 \mathrm{~V}$, and TQWI is connected to low QW via a reset pad. In other time, $V_{\mathrm{RG}}=\mathrm{V}_{1}$ and TQWI is always isolated. $V_{\mathrm{RG}}$ will act as a switch on the charging/discharging of TQWI. A square wave voltage $V_{\mathrm{CG}}$, alternating in $0 \mathrm{~V}$ and $V_{2}\left(V_{2}=-0.04 \mathrm{~V}\right.$ is used here as an example) with frequency $f$ is applied to CG (see Fig. 3(a) and (b), top). $V_{\mathrm{CG}}$ provides the voltage for charging TQWI through the CG/TQWI capacitor.

Fig. 3(c) illustrates how one charging/discharging cycle is going on. The corresponding conduction band profile for each charging/discharging step is schematically depicted in Fig. 3(d). Before the charging/discharging cycle, a negative voltage $V_{1}$ is applied to both IG and RG, so that we get an isolated TQWI. The charging/discharging cycle can be divided into four steps. The low QW electron density (conductance) in step $i$ is denoted as $N_{i}\left(\sigma_{i}\right)$. In each step, TQWI is always isolated and TQWI is only connected to low QW in the pulse between Steps IV and I, and Steps II and III, at those times the charges inside TQWI can flow out/in. These four steps are explained next.

I) $V_{\mathrm{CG}}=0 \mathrm{~V}$, and TQWI is neutral.

II) $V_{\mathrm{CG}}=\mathrm{V}_{2}<0$. Since TQWI is floating and electrons cannot flow in/out, TQWI is still neutral. This neutral TQWI means that it will not screen the electric field from CG. And the negative electric field from CG will pass through top QW, reaching low QW and reducing low QW electron density. Therefore, $N_{\text {II }}<N_{\text {I }}$.

III) $V_{\mathrm{CG}}=\mathrm{V}_{2}<0$. Between Steps II and III, a short positive voltage pulse comes into RG, and TQWI is connected to low QW during the pulse and excessive electrons flow out to low QW, leaving TQWI positively charged and screening the negative electric field from CG. As a result, for Step III, almost no electric field from CG reaches low QW. Therefore, $N_{\text {III }}>N_{\text {II }}$.

IV) $V_{\mathrm{CG}}=0 \mathrm{~V}$. TQWI is positively charged. Due to the capacitive coupling between TQWI and low QW, electron accumulates in low QW. Therefore, $N_{\text {IV }}>N_{\text {III }}$.

After (IV), by a short positive voltage pulse in RG, reset channel turns ON shortly, letting electrons flowing into TQWI during pulse and neutralize TQWI. As a result, electrons accumulated in low QW disappear. The situation returns to (I), starting a next cycle. And, we have $N_{\mathrm{IV}}>N_{\mathrm{I}}$.

From the previous discussion we have $N_{\mathrm{IV}}>N_{\mathrm{I}} \approx N_{\mathrm{III}}>N_{\mathrm{II}}$, as what schematically illustrated in Fig. 3(c). If the mobility in low QW is assumed to be constant, we will have $\sigma_{\mathrm{IV}}>\sigma_{\mathrm{I}} \approx \sigma_{\text {III }}>$ $\sigma_{\text {II }}$. This is just what we observe in CSIP with "Conductance Increase" [see Fig. 3(a)] - the trivial case. The emphasis is Step 
(a)

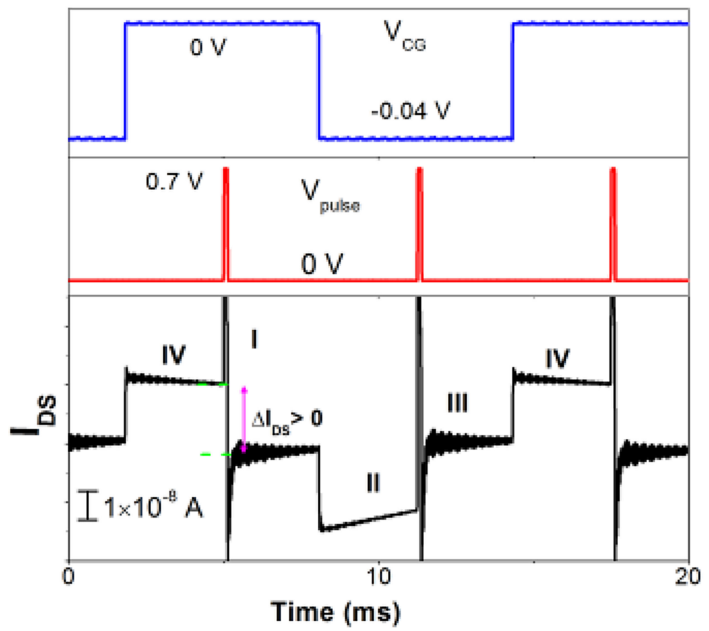

(c)

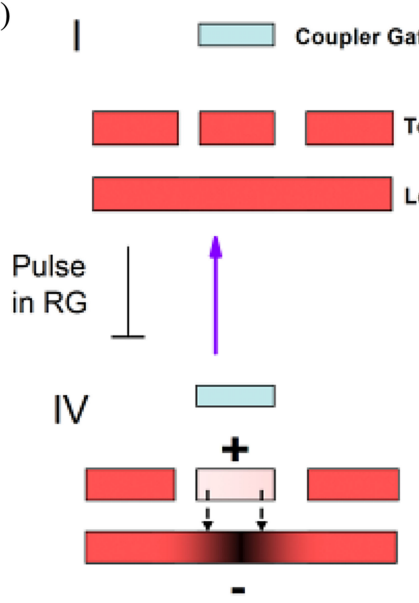

(d)
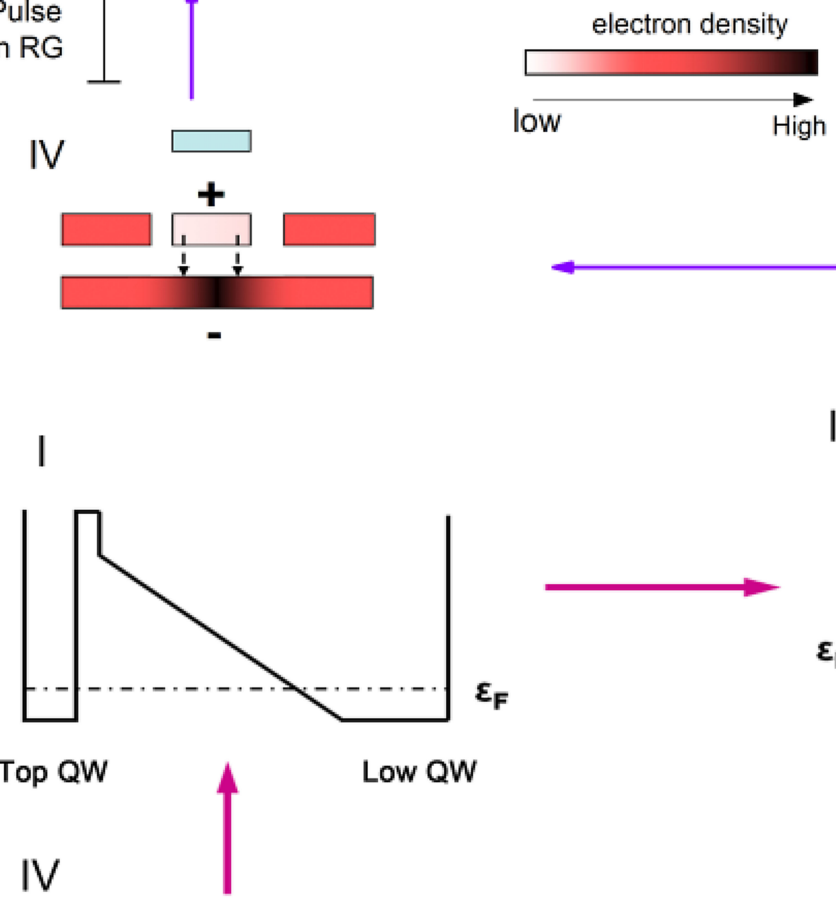

(b)
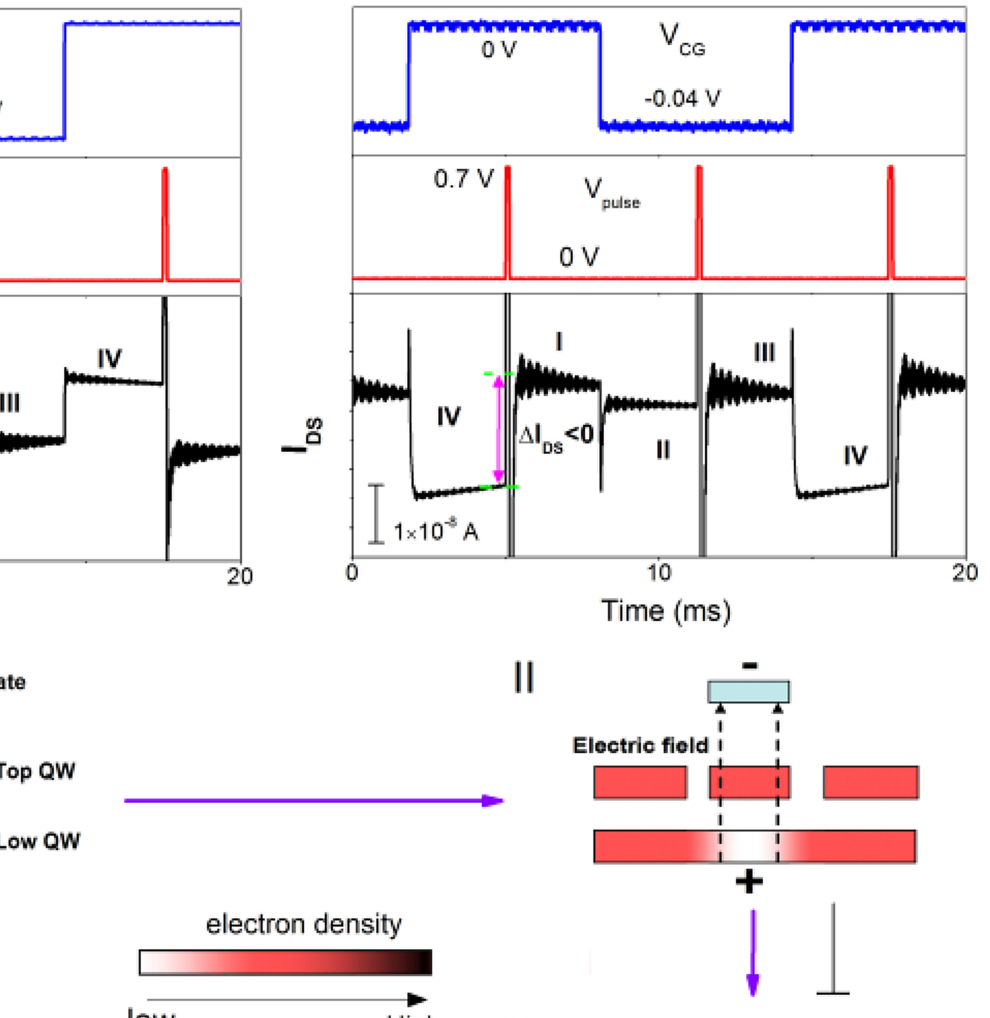

II

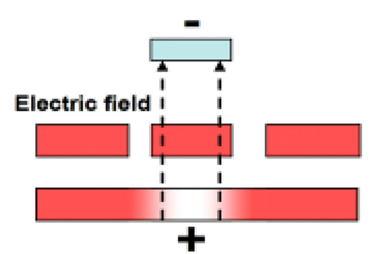

III

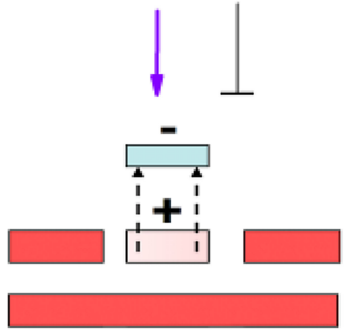

II

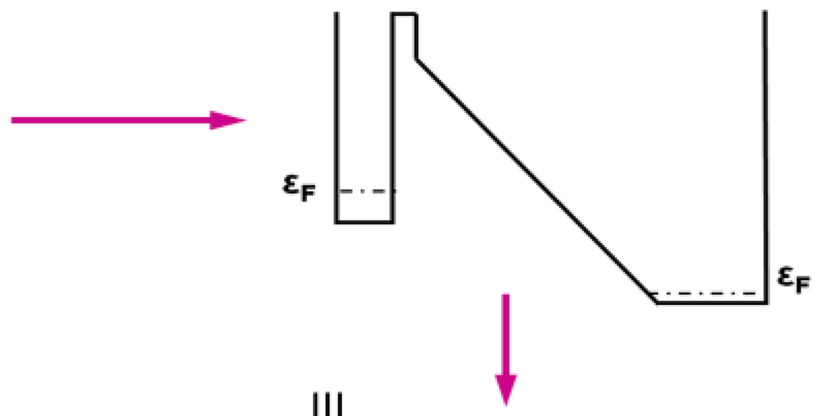

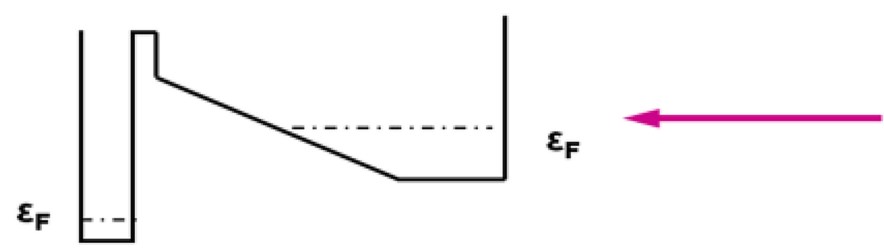

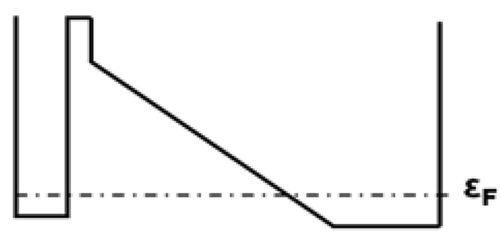

Fig. 3. Time traces of source-drain current $I_{\mathrm{DS}}$ of low QW in the "capacitive charging" measurement: (a) Wafer A with "Conductance Increase"; (b) Wafer B with "Conductance Decrease." The current change between Steps IV and I is figured out. The time evolution of $I_{\mathrm{DS}}$ is monitored by observing the AC component of $\mathrm{I}_{\mathrm{DS}}$ using a four-channel digital oscilloscope (Tektronix, Inc.). The square wave in a coupler gate (frequency $f, f=80 \mathrm{~Hz}$ here) and pulse in a reset gate (frequency $2 f, 2 f=160 \mathrm{~Hz}$ here) are both provided by a multifunction generator (NF Corporation, WAVE FACTORY). (c) Schematic illustration of one cycle of the "capacitive charging" method. A color bar indicating electron density is given in the center. (d) Conduction band profile corresponding to the charging cycle depicted in (c). Note in (a) and (b), the indicated $\Delta I_{\mathrm{DS}}$ values can be different from those $\Delta I_{\mathrm{DS}}$ values in Fig. 2. Because of the charge amount in TQWI, the amount induced by capacitive charging and by photon charging can be different. 
IV which is a mimic of CSIP after light-induced charging, e.g., TQWI with charges and $V_{\mathrm{CG}}=0 \mathrm{~V}$. Compared with CSIP without charging, namely Step I, we are surprised that, under capacitive charging, $\sigma_{\mathrm{IV}}<\sigma_{\mathrm{I}}$ (in contrast with $\sigma_{\mathrm{IV}}>\sigma_{\mathrm{I}}$ in "Conductance Increase") happens for CSIP with "Conductance Decrease" in Fig. 3(b), consistent with the photon-charging results in Fig. 2(b).

This $\sigma_{\mathrm{IV}}<\sigma_{\mathrm{I}}$ is a direct manifestation that, even for CSIP showing "Conductance Decrease," it is not necessary to invoke a negatively charged TQWI to reduce low QW conductance, because positively charged TQWI can do the same job. With a simple knowledge of electrostatic field Gauss's law, positively charged TQWI must enhance low QW electron density. Therefore, "Conductance Decrease" should be mainly attributed to a significant decrease of electron mobility in low QW, rather than an anomalously reversed electron moving into TQWI. Then, the "top-to-low" electron transfer interpretation still holds true.

\section{SUMMARY}

The origin behind this mobility reduction is not very clear yet. And it can be some wafer specified properties, like the ad hoc impurity scatter configuration (which varies notably due to the potential modified by charge presence). One possible candidate is charged donors $D X^{-}-d^{+}\left(D X^{-}\right.$: "deep" states associated with donors; $d^{+}$: "shallow" donors) in the $\mathrm{Al}_{0.3} \mathrm{Ga}_{0.7}$ As material system [10], whose spatial correlation can influence electron mobility and subject to electric field subtly. Photon-induced charges may disturb their correlation and reduce the electron mobility, so result in "Conductance Decrease."

Another attractive topic on the mobility issue in double QWs is the "resistance resonance" observed two decades ago [11]. In this phenomenon, the mobility difference between two QWs, combined with the gate-controlled symmetry of potential profile, can produce some interesting mobility variation effects, which are deeply rooted in the nonlocal nature of quantum mechanical wave function.

However, this highly profound mechanism is only dominant in very closely spaced double QWs, to fulfill the required strong inter-QW tunneling. Or in other words, the ground QW state electron wave function must distribute in both QWs, not only in one certain QW. In contrast, CSIP is just the latter case with the extremely large inter-QW distance $\sim 100 \mathrm{~nm}$ which localizes electrons to certain QW. Therefore, "resistance resonance" can be ruled out.

We notice that "Conductance Decrease" device has a higher low QW electron mobility $\mu_{\mathrm{L}}=9.0 \times 10^{4} \mathrm{~cm}^{2} \mathrm{~V}^{-1} \mathrm{~s}^{-1}$ than "Conductance Increase" device $\mu_{\mathrm{L}}=5.0 \times 10^{4} \mathrm{~cm}^{2} \mathrm{~V}^{-1} \mathrm{~s}^{-1}$. It is reasonable to speculate that higher mobility low QW is more sensitive to scatter configuration in the AlGaAs donor layer that is disturbed by charges in TQWI, so that mobility decrease effect becomes more observable. This high mobility possibly required by "Conductance Decrease" also explains why only a few CSIPs display photon response as "Conductance Decrease," while "Conductance Increase" is the majority. Anyway, mobility constant assumption can be violated in CSIP.

\section{REFERENCES}

[1] S. Komiyama, "Single-photon detectors in the terahertz range," IEEE J. Sel. Topics Quantum Electron., vol. 17, no. 1, pp. 54-66, Jan. 2011

[2] O. Astavief, S. Komiyama, T. Kutsuwa, V. Antonov, Y. Kawaguchi, and K. Hirakawa, "Single-photon detector in the microwave range," Appl. Phys. Lett., vol. 80, pp. 4250-4252, 2002.

[3] Z. An, J. C. Chen, T. Ueda, K. Hirakawa, and S. Komiyama, "Infrared phototransistor using capacitively coupled two-dimensional electron gas layers," Appl. Phys. Lett., vol. 86, pp. 172106-1-172106-3, 2005.

[4] Z. An, J. C. Chen, T. Ueda, K. Hirakawa, and S. Komiyama, "A sensitive double quantum well mid-infrared phototransistor," J. Appl. Phys., vol. 100, pp. 044509-1-044509-7, 2006.

[5] Z. An, T. Ueda, S. Komiyama, and K. Hirakawa, "Metastable excited states of a closed quantum dot with high sensitivity to infrared photons," Phys. Rev. B, vol. 75, pp. 085417-1-085417-7, 2007.

[6] Z. An, T. Ueda, S. Komiyama, and K. Hirakawa, "Reset operation of quantum well infrared phototransistors," IEEE Trans. Electron Devices, vol. 54, no. 7, pp. 1776-1780, Jul. 2007.

[7] Z. Wang, S. Komiyama, T. Ueda, and N. Nagai, "A modified scheme of charge sensitive infrared phototransistor," Appl. Phys. Lett., vol. 95, pp. 022112-1-022112-3, 2009.

[8] P. Nickels, S. Matsuda, T. Ueda, Z. An, and S. Komiyama, "Metal hole arrays as resonant photo-coupler for charge sensitive infrared phototransistors," IEEE J. Quantum Electron., vol. 46, pp. 384-390, Mar. 2010.

[9] C. Sirtori, F. Capasso, J. Faist, and S. Scandolo, "Nonparabolicity and a sum rule associated with bound-to-bound and bound-to-continuum intersubband transitions in quantum wells," Phys. Rev. B, vol. 50, no. 3, pp. 1760-1778, 1994.

[10] E. Buks, M. Heiblum, and H. Shtrikman, "Correlated charged donors and strong mobility enhancement in a two-dimensional electron gas," Phys. Rev. B, vol. 49, pp. 14790-14793, 1994.

[11] A. Palevski, F. Beltram, F. Capasso, L. Pfeiffer, and K. W. West, "Resistance resonance in coupled potential wells," Phys. Rev. Lett., vol. 65, pp. 1929-1932, 1990.

Ting-Ting Kang received the Ph.D. degrees in material physics and chemistry from the Institute of Semiconductors, Chinese Academy of Sciences, Beijing, China, in 2007.

$\mathrm{He}$ is currently with the Shanghai Institute of Technical Physics, Chinese Academy of Sciences, Beijing, China. From 2009 to 2011, he was a Postdoc Researcher in The University of Tokyo, Tokyo, Japan. His research interests include infrared/terahertz detector and quantum transport of low-dimension system.

Susumu Komiyama received the B.S., M.S., and Ph.D. degrees, in 1971, 1973 , and 1976, respectively, all from the University of Tokyo, Tokyo, Japan.

From 1979 to 1981, he was with Hamburg University, Harburg, Germany. During 1976-1982, at the early stage of his career, he was involved in the research on nonlinear transport phenomena and discovered streaming motion and population inversion of hot carriers in ionic crystals and in semiconductors Since 1982, he has been a Faculty Member at the Department of Basic Science, University of Tokyo, where he has been involved in research on quantum Hall effects of 2-D electron gas systems and semiconductor quantum dots. He is a pioneer of developing p-type germanium terahertz lasers (1982-1992) as well as single-terahertz photon detectors based on quantum dots (2000) and CSIPs (2005). He is currently involved in research on establishing passive terahertz microscopy. 
Takeji Ueda received the B.E. and M.E. degrees in applied chemistry from Okayama University, Okayama, Japan, in 1996 and 1998, respectively, and the Ph.D. degree in physics in 2009 from the University of Tokyo, Tokyo, Japan.

He promoted research and development of surface cleaning technology in the semiconductor industry for four years (1998-2002). Through the work, he was interested in developing combined technologies of many different scientific fields. In 2003, he joined the University of Tokyo as a Japan Science and Technology Researcher. He has devoted himself to developing a terahertz photon detector by using quantum nanostructures.

Shi-Wei Lin is currently working toward the Ph.D. degree at the Department of Electronics Engineering, National Chiao Tung University, Hsinchu, Taiwan.
Sheng-Di Lin received the B.S. and M.S. degrees in physics from the National Taiwan University, Taipei, Taiwan, in 1992 and 1994, respectively. After two-year ministry service, he received the Ph.D. degree from the Institute of Electronics Engineering, National Chiao Tung University, Hsinchu, Taiwan, in 2002, where he was involved in metal-semiconductor-metal photodetectors and MBE growth of low-dimensional self-assembled nanostructures.

During 2002-2005, he was a Postdoctoral Research Associate in Cavendish Laboratory, Cambridge University, U.K., where he was involved in focused-ion beam integrated MBE system to fabricate single photon devices. He joined National Chiao Tung University as a faculty member of Department of Electronics Engineering in July 2005. 